\title{
RESULTANT OPERATORS OF A PAIR OF ANALYTIC FUNCTIONS
}

\author{
I. C. GOHBERG AND L. E. LERER
}

\begin{abstract}
The well-known results on resultant of polynomials and its continuous analogue is generalized for some classes of analytic functions.
\end{abstract}

0. Introduction. Let $\lambda_{j}(j=1,2, \ldots, \nu)$ denote the distinct common zeroes of the quasi-polynomials $A_{n}(z)=a_{0}+a_{1} z+\cdots+a_{n} z^{n}$ and $B_{-m}(z)=b_{0}$ $+b_{-1} z^{-1}+\cdots+b_{-m} z^{-m}$ with complex coefficients and let $r_{j}$ be the common multiplicity of the zero $\lambda_{j}$.

The following result is well known (see [2]):

The vectors $\left\{C_{p+k, p} \lambda_{j}^{-(p+k)}\right\}_{k=-n}^{m-1}\left(j=1,2, \ldots, v ; p=0,1, \ldots, r_{j}-1\right)^{1}$ form a basis for the kernel of the resultant matrix:

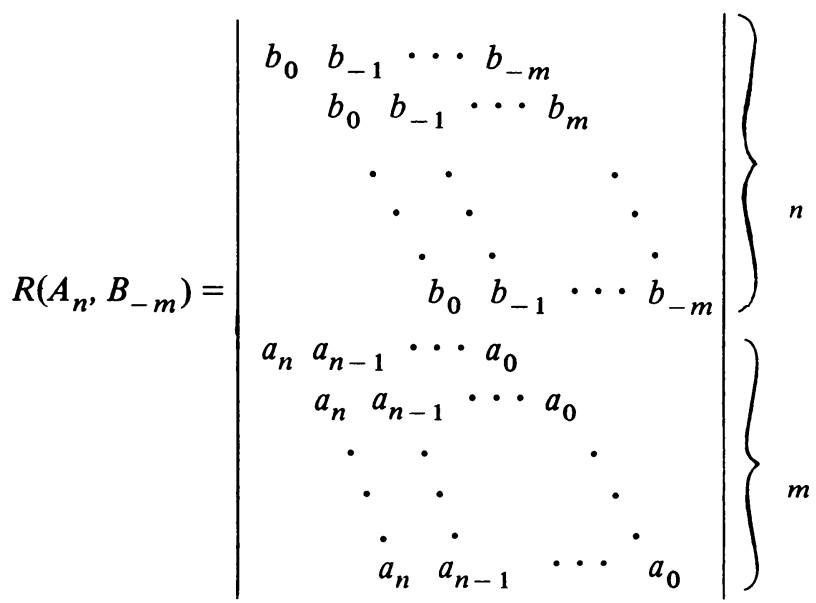

In particular, dim $\operatorname{Ker} R\left(A_{n}, B_{-m}\right)=\sum_{j=1}^{\nu} r_{j}$.

The main aim of the present paper is to extend the above result and its continuous analogue (see [3]) to some classes of analytic functions.

It is natural to expect that in such an extension some kind of a linear operator acting on a suitable infinite dimensional Banach space will play the role of the matrix (1). It will turn out that the choice of a suitable Banach

Received by the editors April 11, 1977 and, in revised form, December 15, 1977.

AMS (MOS) subject classifications (1970). Primary 47B35, 45E10; Secondary 30A08, 30A88.

Key words and phrases. Common zeroes, resultant matrix, Wiener-Hopf pair operator, factorization.

' The numbers $C_{m, p}$ are defined by: $C_{m, 0}=1, C_{m, p}=(m(m-1) \cdot \ldots \cdot(m-p+1)) /(1$, $2 \cdot \ldots \cdot p)$, where $m$ is an arbitrary integer and $p$ is a positive integer. 
space is of great importance for the final statements.

In the first section of the paper we restrict ourselves to the case when the two given functions $A(z)$ and $B(z)$ are represented by series which converge absolutely on the closure $\mathrm{Cl} V_{R}$ of the annulus $V_{R}=\left\{z \in \mathbf{C}^{1}\left|R^{-1}<\right| z \mid<\right.$ $R$ \}:

$$
A(z)=\sum_{j=-\infty}^{\infty} a_{j} z^{j} ; \quad B(z)=\sum_{j=-\infty}^{\infty} b_{j} z^{j} \quad\left(z \in \mathrm{Cl} V_{R}\right) .
$$

Under some conditions the role of the resultant matrix (1) will be played by an operator of the form

$$
W(A, B)=\left|\begin{array}{ccccccccc}
\cdots & & & & & & & & \\
\cdots & b_{1} & b_{0} & b_{-1} & \cdots & & \\
& \cdots & b_{1} & b_{0} & b_{-1} & \ldots & \\
& & \cdots & a_{1} & a_{0} & a_{-1} & \ldots & \\
& & & & \cdots & a_{1} & a_{0} & a_{1} & \ldots
\end{array}\right|
$$

acting on a suitable Banach space of sequences $\xi=\left\{\xi_{j}\right\}_{j=-\infty}^{\infty}$. The choice of this Banach space is important even in the polynomial case when $A=A_{n}$ and $B=B_{-m}$. A simple analysis of the matrix (3) shows that the vectors $\phi_{j p}=$ $\left\{C_{p+k, k} \lambda_{j}^{-(p+k)}\right\}_{k=-\infty}^{\infty}$ form a basis of the subspace $\operatorname{Ker} W\left(A_{n}, B_{-m}\right)$ in the space $l_{1}\left(R_{0}\right)=\left\{\xi=\left\{\xi_{j}\right\}_{j=-\infty}^{\infty}\left|\sum_{j=-\infty}^{\infty} R_{0}^{-|j|}\right| \xi_{j} \mid<\infty\right\}$ if and only if all the zeroes $\lambda_{j}(j=1,2, \ldots, \nu)$ lie in the annulus $V_{R_{0}}$. So in the polynomial case the operator $W\left(A_{n}, B_{-m}\right)$ considered on the space $l_{1}\left(R_{0}\right)$ actually plays the role of the resultant matrix. But in the general case to obtain a complete analogy with the polynomial case we have to make a preliminary shift to the right or to the left of the upper half of the matrix $W(A, B)$ with respect to the lower one. The size of this shift depends on the winding numbers of the functions $A(z)$ and $B(z)$.

The exact statements of all these results are presented in $\S 1$. In the next section we consider the continuous analogues of these results. Here some new surprises appear. In $\$ 3$ we present the main steps of the proofs and in the last section we give an example of applications. All of these results admit a generalization to the matrix-valued case in two directions accordingly to the papers [2], [3] and [4]. These generalizations as well as a generalization for more general domains will appear elsewhere.

1. Functions holomorphic in an annulus. In this section we consider the case when the functions $A(z)$ and $B(z)$ are represented by the series (2) which converge absolutely in the closed annulus $\mathrm{Cl} V_{R}$. We assume that the functions have no zeroes on the boundary of the annulus: 


$$
A(z) \neq 0 ; \quad B(z) \neq 0 \quad\left(|z|=R ;|z|=R^{-1}\right) .
$$

Let us introduce some notations. For a continuous function $F(z)$ which does not vanish on the circle $C_{\rho}=\left\{z \in C^{1}|| z \mid=\rho\right\}$ we denote by $\kappa_{F}(\rho)$ its winding number on $C_{\rho}$, that is $\kappa_{F}(\rho)=(2 \pi)^{-1}\left[\arg F\left(\rho e^{i \phi}\right)\right]_{\phi=0}^{2 \pi}$, where [ $]_{0}^{2 \pi}$ denotes the increment of the function on the segment $[0 ; 2 \pi]$.

Let $E$ be one of the Banach spaces $l_{p}(p \geqslant 1), c, c^{0}$ or $m$ of two-sided sequences $\phi=\left\{\phi_{j}\right\}_{j=-\infty}^{\infty}$. By $E(R)$ we denote the Banach space $E(R)=\{\phi$ $\left.=\left\{\phi_{j}\right\}_{j=-\infty}^{\infty} \mid \phi_{R}=\left\{R^{-|j|} \phi_{j}\right\}_{j=-\infty}^{\infty} \in E\right\}$, with the norm $|\phi|=\left|\phi_{R}\right|_{E}$. In the spaces $E(R)$ we consider the discrete Wiener-Hopf pair operator $W(A, B)$, which is defined on $E(R)$ by $W(A, B) \phi=\left\{\psi_{j}\right\}_{j=-\infty}^{\infty}$, where

$$
\psi_{j}= \begin{cases}\sum_{k=-\infty}^{\infty} a_{j-k} \phi_{k}, & \text { if } j \geqslant 0 \\ \sum_{k=-\infty}^{\infty} b_{j-k} \phi_{k}, & \text { if } j<0\end{cases}
$$

It is well known [1] that the operator $W(A, B)$ is a Fredholm operator in each of the spaces $E(R)$ and that its index $\kappa(W(A, B))=\kappa_{B}(R)-\kappa_{A}\left(R^{-1}\right)$.

We now introduce the shifted operators $W_{l}(A, B)=W\left(A, z^{-l} B\right)(l=0$, $\pm 1, \ldots)$. One can easily check that the following proposition holds.

Proposition 1. The subspaces $\operatorname{Ker} W_{l}(A, B)$ form a descending sequence. There exists an integer $l_{s}$ such that $\operatorname{Ker} W_{l}(A, B)=\operatorname{Ker} W_{l_{s}}(A, B)$ for $l \geqslant l_{s}$ and if $l_{1}<l_{2} \leqslant l_{s}$, then $\operatorname{dim} \operatorname{Ker} W_{l_{1}}(A, B)>\operatorname{dim} \operatorname{Ker} W_{l_{2}}(A, B)$.

The integer $l_{s}$ will be called the index of stabilization of the pair of functions $A(z)$ and $B(z)$. We shall call the operators $W_{l}(A, B)$ with $l \geqslant l_{s}$ the resultant operators. The last definition is justified by the following theorem.

THEOREM 2. Let $A(z)$ and $B(z)$ be two functions of the form (2) which satisfy conditions (4). Let $z_{j}(j=1,2, \ldots, \nu)$ be all the distinct common zeroes of $A(z)$ and $B(z)$ which lie in $V_{R}$ and $r_{j}(j=1,2, \ldots, v)$ be their common multiplicities.

Then the index of stabilization can be calculated as follows

$$
l_{s}=\kappa_{B}(R)-\kappa_{A}\left(R^{-1}\right)-\sum_{j=1}^{\nu} r_{j}
$$

and for every $l \geqslant l_{s}$ the vectors

$$
\phi_{j p}=\left\{C_{p+k, p} \lambda_{j}^{-(p+k)}\right\}_{k=-\infty}^{\infty} \quad\left(j=1,2, \ldots, \nu ; p=0,1, \ldots, r_{j}-1\right)
$$

form $a$ basis of the subspace $\operatorname{Ker} W_{l}(A, B)$. In particular,

$$
\operatorname{dim} \operatorname{Ker} W_{l}(A, B)=\sum_{j=1}^{\nu} r_{j} .
$$

Note that $l^{\prime}=\kappa(W(A, B)) \leqslant l_{s}$ and therefore each operator $W_{l}(A, B)$ with 
$l>l^{\prime}$ is a resultant operator. It is remarkable that the integer $l^{\prime}$ does not depend on the number of common zeroes of $A(z)$ and $B(z)$.

2. The continuous case. To begin with let us introduce some notations.

Given a Banach space $E$ of functions defined on the real axis $R^{1}$ and given a continuous function $\sigma(t)$ which does not vanish on that axis. Let us agree to denote by $\sigma(t) E$ the Banach space of all functions $f(t)\left(t \in R^{1}\right)$ such that $\sigma^{-1}(t) f(t) \in E$ with the norm $|f|=\left|\sigma^{-1} f\right|_{E}$. If $a<b$ are two real numbers, we set $\Gamma_{a}=\left\{z \in \mathbf{C}^{\mathbf{l}} \mid \operatorname{Im} z=a\right\}$ and $\Pi(a, b)=\left\{z \in \mathbf{C}^{\mathbf{l}} \mid a<\operatorname{Im} z<b\right\}$.

In this section we consider the case when the functions $A(z)$ and $B(z)$ are represented by Fourier transforms in the strip $\Pi(-h, h)(h>0)$ :

$$
\begin{aligned}
A(z)=1+\int_{-\infty}^{\infty} a(t) e^{i z t} d t ; \quad B(z)=1+\int_{-\infty}^{\infty} b(t) e^{i z t} d t & \\
& (z \in \mathrm{Cl} \Pi(-h, h)) .
\end{aligned}
$$

As in $\S 1$ we assume that the functions $A(z)$ and $B(z)$ have no zeroes on the boundary of the domain:

$$
A(z) \neq 0 ; \quad B(z) \neq 0 \quad\left(z \in \Gamma_{h} \cup \Gamma_{-h}\right) .
$$

For two such functions one can construct a complete analogue of the operators $W_{l}(A, B)$ which corresponds to the "discrete" shift. The notion of the index of stabilization is well defined for these analogues and one can obtain results which are similar to Proposition 1 and Theorem 2. However, we present here a quite different class of resultant operators which are associated with a continuous shift. Let us denote by $W^{\varepsilon}(A, B)(\varepsilon>0)$ the following operator acting on $e^{h|t|} E$ :

$$
\left(W^{\varepsilon}(A, B) \phi\right)(t)=\left\{\begin{array}{l}
\phi(t)+\int_{-\infty}^{\infty} a(t-s) \phi(s) d s \quad(t \geqslant 0), \\
\phi(t+\varepsilon)+\int_{-\infty}^{\infty} b(t+\varepsilon-s) \phi(s) d s \quad(t<0) .
\end{array}\right.
$$

It turns out that these operators may play the role of the resultant operators. More precisely, the following result holds.

THEOREM 3. Let $A(z)$ and $B(z)$ be two functions of the form (5) which satisfy conditions (6). Let $z_{j}(j=1,2, \ldots, \nu)$ be all the distinct common zeroes of $A(z)$ and $B(z)$ in $\Pi(-h, h)$ and let $r_{j}(j=1,2, \ldots, \nu)$ be their multiplicities.

Then for every $\varepsilon>0$ the functions

$$
\phi_{j p}(t)=t^{p} \exp \left(-i z_{j} t\right) \quad\left(j=1,2, \ldots, \nu ; p=0,1, \ldots, r_{j}-1\right)
$$

form a basis of the subspace $\operatorname{Ker} W^{\varepsilon}(A, B)$. In particular,

$$
\operatorname{dim} \operatorname{Ker} W^{\varepsilon}(A, B)=\sum_{j=1}^{\nu} r_{j}
$$

3. The proof. We shall present here the proof of Theorem 3 only.

First of all we introduce the following notation. Let $K(h)(h>0)$ denote 
the ring of all functions $A(\zeta)$ which are defined on the contour $\Gamma_{h} \cup \Gamma_{-h}$ as follows

$$
A(\zeta)=c+\int_{-\infty}^{\infty} e^{i \zeta t} a(t) d t \quad\left(\zeta \in \Gamma_{h} \cup \Gamma_{-h}\right)
$$

where $a(t) \in e^{-h|t|} L_{1}(-\infty, \infty)$ and $c$ is a complex constant. It is clear that every function $A(\zeta) \in K(h)$ can be extended to a function $A(z)$ which is holomorphic in the strip $\Pi(-h, h)$ and continuous on its closure. By $K^{+}(h)$ we denote the subring of $K(h)$ consisting of all functions $A(\zeta) \in K(h)$ with $a(t)=0$ for $t<0$. Obviously, each function $A(\zeta) \in K^{+}(h)$ admits an extension which is holomorphic in $\Pi(-h, \infty)$ and continuous on $\mathrm{Cl} \Pi(-k, \infty)$. The symbol $K^{-}(h)$ has an analogous meaning. Recall that the winding number of a function $A(\zeta)$, which is continuous and different from zero on the line $\Gamma_{b}$, is defined as the integer

$$
\kappa_{A}(b)=(2 \pi)^{-1}[\arg A(\lambda+i b)]_{\lambda=-\infty}^{\infty} .
$$

Now let $A(\zeta) \in K(h)$ and suppose that $A(\zeta) \neq 0\left(\zeta \in \Gamma_{h} \cup \Gamma_{-h}\right)$. We denote by $\lambda_{j}(j=1,2, \ldots, \alpha)$ all the zeroes of $A(z)$ in the strip $\Pi(-h, h)$ counting multiplicities, and we define a rational function $P_{A}(z)$ which corresponds to $A(\zeta)$ as follows:

$$
P_{A}(z)=[(z+\theta) /(z-\theta)]^{\kappa} \prod_{j=1}^{\alpha}\left[\left(z-\lambda_{j}\right) /(z-\theta)\right],
$$

where $\kappa=\kappa_{A}(h)$ and $\theta$ is a fixed point in $\Pi(-\infty, h)$. Let us recall that by $\mathbf{M}$. G. Krein's results [5] each function $A(\zeta) \in K(h)$ which is nonzero on $\Gamma_{h} \cup$ $\Gamma_{-h}$ admits a factorization of the form $A(z)=A_{+}(z) P_{A}(z) A_{-}(z)$, where $\left(A_{ \pm}\right)^{ \pm 1} \in K^{ \pm}(h)$.

Proof of Theorem 3. At first we shall establish that

$$
\operatorname{Ker} W^{\varepsilon}(A, B)=\operatorname{Ker} W(A) \cap \operatorname{Ker} W(B)
$$

for every $\varepsilon>0$, where the operator $W(A)$ is defined on $e^{h|x|} E$ as

$$
(W(A) \phi)(t)=\phi(t)+\int_{-\infty}^{\infty} a(t-s) \phi(s) d s \quad(-\infty<t<\infty) .
$$

The relation $\operatorname{Ker} W^{\varepsilon}(A, B) \supset \operatorname{Ker} W(A) \cap \operatorname{Ker} W(B)$ is self-evident and therefore it remains only to prove the converse relation. Let $\phi(t) \in$ $\operatorname{Ker} W^{\varepsilon}(A, B)$. Because of Theorem 1.1 in the Appendix of [1] we may assume that $\phi(t) \in e^{h|t|} L_{1}(-\infty, \infty)$. Introduce two functions

$$
\begin{aligned}
& f(t)= \begin{cases}0 & (t>0), \\
\phi(t)+\int_{-\infty}^{\infty} a(t-s) \phi(s) d s & (t<0) ;\end{cases} \\
& g(t)= \begin{cases}\phi(t)+\int_{-\infty}^{\infty} b(t-s) \phi(s) d s & (t>\varepsilon), \\
0 & (t<\varepsilon) .\end{cases}
\end{aligned}
$$


It is easily seen that $f \in e^{-h t} L_{1}(-\infty, \infty)$ and $g \in e^{h t} L_{1}(-\infty, \infty)$ and therefore that the function $F(z)=\int_{-\infty}^{0} f(t) e^{i z t} d t$ (accordingly $G(z)=$ $\left.\int_{e}^{\infty} g(t) e^{i z t} d t\right)$ admits an extension which is holomorphic in $\Pi(-\infty,-h)$ (accordingly $\Pi(h, \infty))$ and continuous on its closure. The condition $\phi \in$ $\operatorname{Ker} W^{\ell}(A, B)$ can be rewritten as the following system of equations

$$
\left\{\begin{array}{l}
\phi(t)+\int_{-\infty}^{\infty} a(t-s) \phi(s) d s=f(t) \\
\phi(t)+\int_{-\infty}^{\infty} b(t-s) \phi(s) d s=g(t)
\end{array} \quad(-\infty<t<\infty) .\right.
$$

Let $P$ be the projection defined on $e^{h|t|} E$ by the rule: $(P \phi)(t)=\phi(t)$, if $t \geqslant 0$, and $(P \phi)(t)=0$, if $t<0$, and let $Q=I-P$. Then the first equation of (8) may be written as

$$
\begin{aligned}
(P \phi)(t)+\int_{-\infty}^{\infty} a(t-s)(P \phi)(s) d s & \\
& \left.=-(Q \phi)(t)-\int_{-\infty}^{\infty} a(t-s)(Q \phi)(s) d s+f(t) \quad \stackrel{\text { def }}{=} \omega_{1}(t)\right) .
\end{aligned}
$$

It is not difficult to show that $\omega_{1}(t) \in e^{-h|t|} L_{1}(-\infty, \infty)$ and therefore that $\Omega_{1}(z)=\int_{-\infty}^{\infty} \omega_{1}(t) e^{i z t} d t \in K(h)$. Multiplying (9) by $e^{-h t}$ or $e^{h t}$ and writing in both cases the corresponding Fourier transform, we obtain the relations

$$
\begin{gathered}
A(\zeta) \Phi_{+}(\zeta)=\Omega_{1}(\zeta) \quad\left(\zeta \in \Gamma_{h}\right) ; \\
A(\zeta) \Phi_{-}(\zeta)_{-} F(\zeta)=-\Omega_{1}(\zeta) \quad\left(\zeta \in \Gamma_{-h}\right),
\end{gathered}
$$

where

$$
\Phi_{+}(\zeta)=\int_{0}^{\infty} \phi(t) e^{i \zeta t} d t \text { and } \Phi_{-}(\zeta)=\int_{-\infty}^{0} \phi(t) e^{i \zeta t} d t .
$$

An analogous procedure applied to the second equation of (8) leads to the equations

$$
\begin{gathered}
B(\zeta) \Phi_{+}(\zeta)+G(\zeta)=\Omega_{2}(\zeta) \quad\left(\zeta \in \Gamma_{h}\right) \\
-B(\zeta) \Phi_{-}(\zeta)=\Omega_{2}(\zeta) \quad\left(\zeta \in \Gamma_{-h}\right)
\end{gathered}
$$

with $\Omega_{2}(\zeta) \in K(h)$. We now define two functions on $\mathrm{Cl} \Pi(-h, h)$ :

$$
\begin{aligned}
X(z) & =[(z-\theta) /(z+\theta)]^{\alpha^{\prime}} P_{B}(z) B_{-}(z) A_{-}^{-1}(z) ; \\
Y(z) & =[(z-\theta) /(z+\theta)]]^{\alpha^{\prime}} P_{A}(z) A_{+}(z) B_{+}^{-1}(z),
\end{aligned}
$$

where $\kappa^{\prime}=\kappa_{B}(-h), \theta \in \Pi(-\infty,-h)$ and $A_{ \pm}, B_{ \pm}, P_{A}, P_{B}$ are the factors of the factorization mentioned above of the functions $A$ and $B$. One can easily check that $X(z) A(z)-Y(z) B(z)=0(z \in \mathrm{Cl} \Pi(-h, h))$. Using this equation we can eliminate the functions $\Phi_{+}$and $\Phi_{-}$from (10)-(11) and obtain the following system. 


$$
\left\{\begin{aligned}
-Y(\zeta) G(\zeta) & =X(\zeta) \Omega_{1}(\zeta)-Y(\zeta) \Omega_{2}(\zeta) & & \left(\zeta \in \Gamma_{h}\right), \\
X(\zeta) F(\zeta) & =X(\zeta) \Omega_{1}(\zeta)-Y(\zeta) \Omega_{2}(\zeta) & & \left(\zeta \in \Gamma_{-h}\right)
\end{aligned}\right.
$$

A simple analysis of (12) shows that the functions $X \Omega_{1}-Y \Omega_{2}, X F$ and $Y G$ can be well extended into the appropriate domains so that the function $R(z)$ defined on $C^{1}$ as

$$
R(z)=\left\{\begin{array}{l}
-Y(z) G(z) \quad(z \in \Pi(h, \infty)), \\
X(z) \Omega_{1}(z)-Y(z) \Omega_{2}(z) \quad(z \in \mathrm{Cl} \Pi(-h, h)), \\
X(z) F(z) \quad(z \in \Pi(-\infty,-h))
\end{array}\right.
$$

is holomorphic in the whole complex plane except perhaps of the point $z=-\theta$. This point may be a pole of a finite order $\left(\leqslant \kappa_{B}(-h)-\kappa_{A}(h)\right)$. In addition, $R(\infty)=0$. Hence, $R(z)=S(z)(z+\theta)^{-m}$, where $S(z)$ is a polynomial with $\operatorname{deg} S \leqslant m-1$. We shall show that in fact $R(z) \equiv 0$. Indeed, the function $G(z)$ can be represented in the form $G(z)=G_{1}(z) e^{i z \varepsilon}$, where

$$
G_{1}(z)=\int_{0}^{\infty} g(t+\varepsilon) e^{i z t} d t \quad(z \in \mathrm{Cl} \Pi(h, \infty)) .
$$

Hence, $S(z)=-Y(z) G_{1}(z) e^{i z \varepsilon}(z+\theta)^{m}$. This equation implies that $S(z) \rightarrow$ 0 if $\operatorname{Im} z \rightarrow \infty$ and therefore $A(z) \equiv 0$ on $\mathbf{C}^{1}$. Hence, $R(z) \equiv 0$ on $\mathbf{C}^{\mathbf{l}}$.

The last equation leads to the equations $F(z)=G(z)=0$, which are equivalent to the following: $f(t)=g(t)=0$. This means obviously that $\phi(t) \in \operatorname{Ker} W(A) \cap \operatorname{Ker} W(B)$ and therefore the relation (7) is proved. Now using Theorem 2.1 of the Appendix of [1], which describes the kernels of the operators $W(A)$, one can easily complete the proof.

REMARK 4. The above proof shows that in the case $a(t)=0(t<0)$ and $b(t)=0(t>0)$ Theorem 3 is valid also, if we assume $\varepsilon=0$.

Indeed, in that case we may set $X=B \in K^{-}(h), Y=A \in K^{+}(h)$ and the function $Y G$ is holomorphic in $\Pi(h, \infty)$ for every $\varepsilon \geqslant 0$.

4. Applications. The results mentioned above may be used, for instance, to find a solution of a system of two equations with two unknowns by an elimination method.

Let us consider, for example, the discrete case. Suppose that the two functions

$$
A(\lambda, \mu)=\sum_{j, k=-\infty}^{\infty} a_{j k} \lambda^{j} \mu^{k} \text { and } B(\lambda, \mu)=\sum_{j, k=-\infty}^{\infty} b_{j k} \lambda^{j} \mu^{k}
$$

are represented as absolutely convergent series in the closed polyannulus $\mathrm{Cl} V_{R} \times V_{R}$ and consider the following system of equations

$$
A(\lambda, \mu)=0, \quad B(\lambda, \mu)=0 .
$$

We assume that the functions $A(\lambda, \mu)$ and $B(\lambda, \mu)$ satisfy the following 
conditions: (a) for some $\mu^{\prime}$ the system (13) has no solutions; (b) $A(\lambda, \mu) \neq 0$, $B(\lambda, \mu) \neq 0$ if $\lambda \in C_{1 / R} \cup C_{R}, \mu \in \mathrm{Cl} V_{R}$.

Rewrite the functions as follows:

$$
A(\lambda, \mu)=\sum_{j=-\infty}^{\infty} A_{j}(\mu) \lambda^{j} \text { and } B(\lambda, \mu)=\sum_{j=-\infty}^{\infty} B_{j}(\mu) \lambda^{j}
$$

and denote by $W_{l}(\mu)$ the operator generated in $l_{1}(R)$ by the matrix

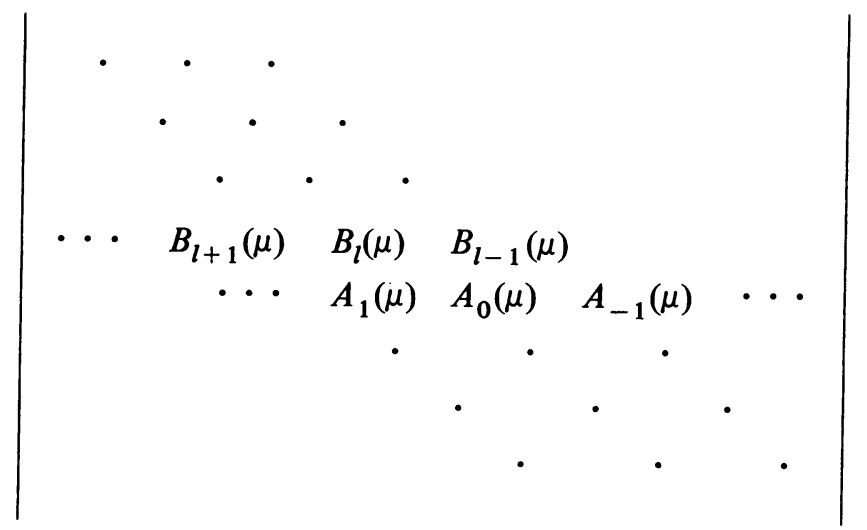

Let $l^{\prime}=\kappa_{B\left(\lambda, \mu^{\prime}\right)}(R)-\kappa_{A\left(\lambda, \mu^{\prime}\right)}\left(R^{-1}\right)$. The operator $W_{l^{\prime}}(\mu)$ is an analytic function of the variable $\mu$ and it is invertible for all $\mu \in V_{R}$ except perhaps a finite set of points $M_{0}$. At these points $\operatorname{dim} \operatorname{Ker} W_{l}\left(\mu_{0}\right)>0\left(\mu_{0} \in M_{0}\right)$. Now it follows from Theorem 2 that the system (13) is solvable if and only if $\mu \in M_{0}$. Setting $\mu=\mu_{0} \in M_{0}$ in (13) we obtain

$$
A\left(\lambda, \mu_{0}\right)=0, \quad B\left(\lambda, \mu_{0}\right)=0 .
$$

Therefore in order to solve the system (13) with two unknowns we have to solve the system (15) with one unknown.

Now change the role of the variables $\lambda$ and $\mu$ and find a finite set of points $\Lambda_{0} \subset V_{R}$ for which the system (13) is solvable. It remains, therefore, to couple points from $\Lambda_{0}$ and $M_{0}$ which satisfy the equations $A\left(\lambda_{0}, \mu_{0}\right)=0, B\left(\lambda_{0}, \mu_{0}\right)=$ 0 .

\section{REFERENCES}

1. I. C. Gohberg and I. A. Feldman, Convolution equations and projection methods of their solution, "Nauka", Moscow, 1971; English transl., Transl. Math. Monographs, vol. 41, Amer. Math. Soc., Providence, R.I., 1974.

2. I. C. Gohberg and G. Heinig, Resultant matrix and its generalization. I. Resultant operator of matrix polynomials, Acta Sci. Math. (Szeged) 37 (1975), Fasc. 1 - 2, pp. 41-61. (Russian)

3. __ Resultant matrix and its generalization. II: Continual analog of resultant matrix, Acta Math. Acad. Sci. Hungar. 28 (1976), 3-4, 189-209. (Russian)

4. I. C. Gohberg and L. E. Lerer, Resultants of matrix polynomials, Bull. Amer. Math. Sci. 82 (1976), 565-567. 
5. M. G. Krein, Integral equations on a half-line with kernel depending upon the difference of the arguments, Uspehi Mat. Nauk 13 (1958), no. 5 (83), 2-120; English transl., Amer. Math. Soc. Transl. (2) 22 (1962), 163-288.

Department of Mathematical Sciences, Tel-Aviv University, Tel-Aviv, Israel

Department of Pure Mathematics, Weizmann Institute of Science, Rehovot, Israel

Department of Mathematics, Techinion, Haifa, Israel 